\title{
Mid-Infrared Dual-Comb Spectroscopy as sensor: Fast and precise quantification of multiple gases
}

\author{
Leonard Nitzsche ${ }^{1}$, Jens Goldschmidt ${ }^{1}$, Jens Kießling ${ }^{1}$, Sebastian Wolf ${ }^{1}$, Frank Kühnemann ${ }^{1}$, Jürgen \\ Wöllenstein ${ }^{1,2}$ \\ ${ }^{1}$ Fraunhofer Institute for Physical Measurement Techniques IPM, Georges-Köhler-Alle 301, \\ $D$-79110 Freiburg, Germany \\ 2 University of Freiburg, Department of Microsystems Engineering IMTEK, \\ Laboratory for Gas Sensors, Georges-Köhler-Allee 102, D-79110 Freiburg, Germany \\ juergen.woellenstein@imtek.uni-freiburg.de
}

\begin{abstract}
Summary:
Dual-comb spectroscopy allows to record broadband transmission spectra fast and precisely. This bridges the gap between classical methods, where Fourier-Transform infrared spectroscopy excels in spectral bandwidth or tunable laser absorptions spectroscopy in sensitivity. Here we present a dualcomb based spectrometer for gas sensing in mid-infrared from $3 \mu \mathrm{m}$ to $5 \mu \mathrm{m}$. Using the spectrometer as a sensor for a mixture of nitrous oxide $\left(\mathrm{N}_{2} \mathrm{O}\right)$ and carbon monoxide (CO) results in 48.3(2) ppm $\mathrm{N}_{2} \mathrm{O}$ and 29.10(7) ppm CO in $10 \mathrm{~s}$. The limits of detection are $90 \mathrm{ppb} \mathrm{N} \mathrm{N}_{2} \mathrm{O}$ and $49 \mathrm{ppb} \mathrm{CO}$ in $25 \mathrm{~s}$.
\end{abstract}

Keywords: dual-comb spectroscopy, spectrometer, mid-infrared, trace gas, process analytics

\begin{abstract}
Motivation
Dual-comb spectroscopy allows to record broadband transmission spectra composed of thousands of equidistant independent spectral elements. Although this method is well established in many laboratories around the world its potential of being used as a sensor for e.g. process analytics is yet not fully developed. This target in mind we design a spectrometer especially suited for the detection of multiple trace gases in a single and short measurement. This is a necessity to first optimize and potentially enable active control over processes where high purity of the educts is needed, e.g. a low carbon monoxide content in hydrogen for fuel cells [1]. It is important for many catalytic reactions as methane synthesis based on nickel catalysts, where hydrogen sulfide poisons the catalyst leading to decreasing efficiency [2], as well. In contrast to being precise, our spectrometer allows fast acquisition rates $(\mathrm{kHz})$ which is important for testing exhaust or optimizing combustion processes. To demonstrate that our spectrometer bridges the gap between fast acquisition rates and high precision measurements we investigate a mixture of nitrous oxide and carbon monoxide, both occurring with concentrations below $50 \mathrm{ppm}$ in the chosen gas matrix.
\end{abstract}

\section{Working principle of the spectrometer}

A single frequency comb can be described as a pulsed laser where its optical spectrum is composed of multiple discrete modes with identical spacing $f_{\mathrm{r}}$. As the comb spectrum covers a broad spectral range, the attenuation caused by a sample, here two gases, can be determined. By superimposing the probing comb with a reference comb with a slightly different mode spacing - often referred to as dual-comb spectroscopy [3] - beatings between pairs of comb modes are generated. From the beat signal - interferogram - the transmission spectrum can be reconstructed in analogy to Fourier-transform infrared spectroscopy (FTIR). No moving parts are required nor does any instrument function bias the recorded spectra. In addition, the high optical powers and beam properties allow using gas cells with longer absorption length or even open-path applications. With the possibility to build such spectrometers robust and space-efficient, it is a promising alternative to classical analytical methods as gas chromatography or FTIR.

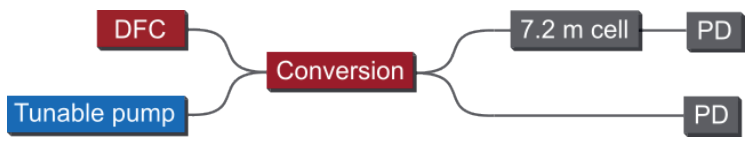

Fig. 1. Hardware modules of the spectrometer. The near-infrared dual-frequency comb (DFC) at $1550 \mathrm{~nm}$ is converted to the mid-infrared. By using a tunable pump from $1.0 \mu \mathrm{m}$ to $1.3 \mathrm{\mu m}$ the converted mid-infrared combs can be positioned in the spectral range from $3 \mu \mathrm{m}$ to $5 \mu \mathrm{m}$. The DFC is split into two branches. One propagates through a multi-reflection flow cell and the other is detected directly serving as reference. 


\section{Experimental setup}

To realize such a spectrometer we make use of a fiber-based dual-comb generator emitting a superimposed dual-frequency comb (DFC) at $1550 \mathrm{~nm}$ further explained in [4]. The mode spacing can be tuned from $0.008 \mathrm{~cm}^{-1}$ to 0.016 $\mathrm{cm}^{-1}$ and the spectral bandwidth can be chosen from $6 \mathrm{~cm}^{-1}$ to $20 \mathrm{~cm}^{-1}$. To reach the MIR we convert the near-infrared DFC via difference frequency generation to the mid-infrared. Combined with a tunable pump laser for this process we are able to reach the spectral region from $3 \mu \mathrm{m}$ to $5 \mu \mathrm{m}$, which is of special interest as many gases show strong and characteristic absorptions in this region. The converted DFC is split into two branches. In the sample branch channel the dual-comb signal passes a multi-pass cell with $7.2 \mathrm{~m}$. The signal from the reference branch is used to normalize the spectra from the sample branch.

\section{Results}

With this configuration we first record a reference spectrum of nitrogen - not absorbing -, fill the cell with a mixture of nitrous oxide $\left(\mathrm{N}_{2} \mathrm{O}\right)$ and carbon monoxide (CO), and record a transmission spectrum of the sample.

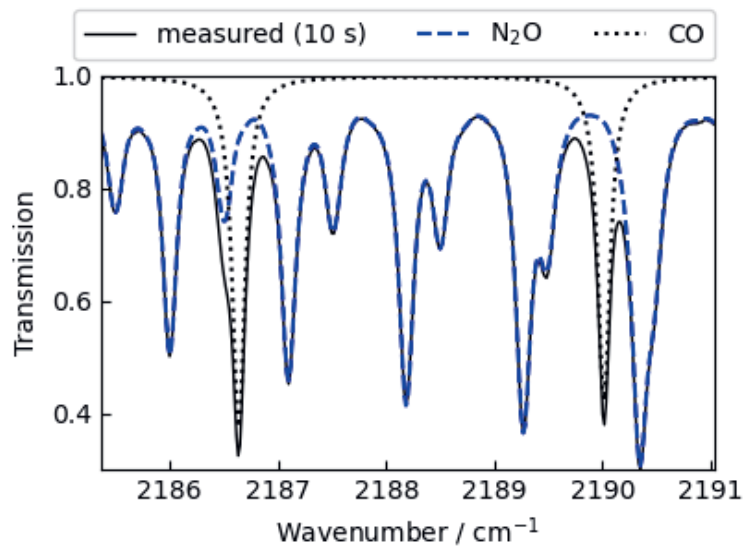

Fig. 2. Measured transmission spectrum of $48.3 \mathrm{ppm}$ nitrous oxide $\left(\mathrm{N}_{2} \mathrm{O}\right)$ and $29.1 \mathrm{ppm}$ carbon monoxide (CO) in synthetic air. For convenience, the dashed lines indicate the contributions from both compounds to the spectrum.

To derive the concentrations of both compounds we fit a simulation using the HITRAN database to the sample spectrum corrected by the reference spectrum. This results in $48.3 \mathrm{ppm}$ $\mathrm{N}_{2} \mathrm{O}$ and $29.10 \mathrm{ppm}$, both with $\pm 3 \%$ uncertainty $(1 \sigma)$, which is in accordance with our expectation and is shown in Fig. 2. Evaluating sample spectra with $10 \mathrm{~Hz}$ acquisition rate, as provided in Fig. 3, shows that after already $25 \mathrm{~s}$ of measurement time the relative uncertainty $(1 \sigma)$ of the determined gas concentration is as low as $0.6 \%$ for $\mathrm{N}_{2} \mathrm{O}$ and $0.5 \%$ for $\mathrm{CO}$, which corresponds to a $90 \mathrm{ppb}$ limit of detection of $\mathrm{N}_{2} \mathrm{O}$ and $49 \mathrm{ppb}$ for $\mathrm{CO}$ likewise.

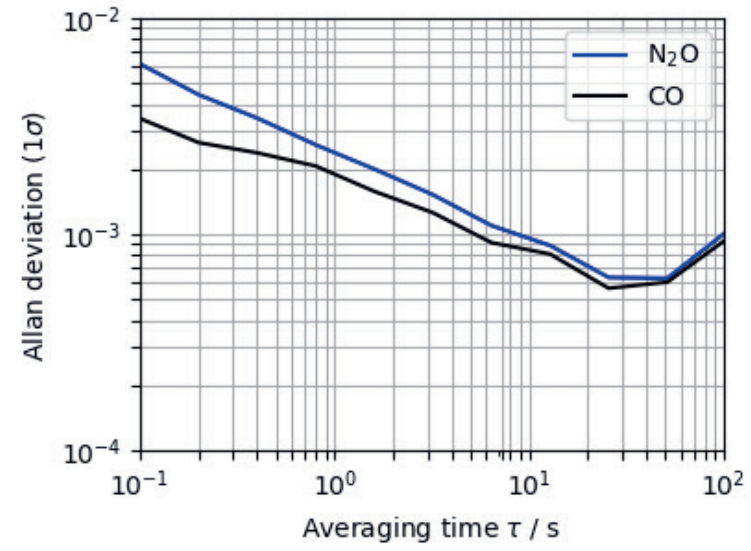

Fig. 3. Allan deviation of determined gas concentrations - $48.3 \mathrm{ppm}$ for $\mathrm{N}_{2} \mathrm{O}$ and $29.1 \mathrm{ppm}$ for $\mathrm{CO}$ - acquired with $10 \mathrm{~Hz}$ rate. The minimum is reached after $25 s$ at $0.6 \%$ for $\mathrm{N}_{2} \mathrm{O}$ and $0.5 \%$ for $\mathrm{CO}$.

\section{References}

[1] V. F. Valdés-López, T. Mason, P. R. Shearing, D. J. L. Brett, Carbon monoxide poisoning and mitigation strategies for polymer electrolyte membrane fueal cells - A review, Progress in Energy and Combustion Science 79, 10084 (2020), doi: doi.org/10.1016/j.pecs.2020.100842

[2] C. H. Bartholomew, Mechanisms of catalyst deactivation, Appl. Catalysis A 212, 17-60 (2001), doi: 10.1016/S0926-860X(00)00843-7

[3] I. Coddington, N. Newbury, W. Swann, Dualcomb spectroscopy, Optica 3, 414-426 (2016); doi: 10.1364/OPTICA.3.000414

[4] L. Nitzsche, J. Kießling, S. Wolf, F. Kühnemann, J. Wöllenstein, Ultra-fast gas spectroscopy with a dual-comb spectrometer, SMSI 2020, 123 - 124, doi: DOI 10.5162/SMSI2020/B4.4

[5] R.V. Kochanov, I.E. Gordon, L.S. Rothman, P. Wcislo, C. Hill, J.S. Wilzewski, HITRAN Application Programming Interface (HAPI): A comprehensive approach to working with spectroscopic data, J. Quant. Spectrosc. Radiat. Transfer 177, 15-30 (2016); doi: 10.1016/j.jqsrt.2016.03.005 